\title{
QOC*: utilizando Design Rationale como ferramenta para gerenciar conhecimento em projetos de software
}

\author{
Marcos Camponogara ${ }^{1}$ \\ Milene Silveira ${ }^{2}$
}

\begin{abstract}
Resumo: Durante o processo de desenvolvimento de um sistema de software, uma grande quantidade de conhecimento é utilizada e produzida como resultado das opções analisadas e das decisões tomadas ao longo do desenvolvimento do projeto. Este conhecimento é valioso, pois reflete as razões que estão por trás das decisões, o que facilita o entendimento dos rumos do projeto e proporciona uma visão global do mesmo. Desta forma, existe a necessidade de se encontrar alternativas para organizar e manter este tipo de conhecimento e então torná-lo um recurso que possa facilitar a continuidade de projetos de software ou então a manutenção de sistemas desenvolvidos. Neste sentido, este artigo apresenta uma pesquisa baseada em Gestão do Conhecimento e Design Rationale, que propõe uma maneira de representar e manter as razões que motivaram a tomada de determinadas decisões em projetos de software, considerando, para isso, uma representação baseada no questionamento e na discussão a respeito das melhores opções para atender questões que surgem durante o desenvolvimento de um projeto.
\end{abstract}

Palavras-chave: Gestão do Conhecimento. Design Rationale. Tomada de decisão.

\footnotetext{
${ }^{1}$ PPGCC/PUCRS, Avenida Ipiranga, 6681, Prédio 32, Porto Alegre, RS - Brasil. \{mcamponogara@gmail.com\}

${ }^{2}$ PPGCC/PUCRS, Avenida Ipiranga, 6681, Prédio 32, Porto Alegre, RS - Brasil. $\{$ milenelinf.pucrs.br $\}$
} 
Abstract: In general, the decision making process in a software development project depends on the people involved in this process and their knowledge of the project. Also, the option analysis process which results in a decision, produces a kind of knowledge that is very important for the project and its future, since this knowledge is about key decisions and their rationale. Thus, understanding the rationale behind a decision provides a powerful way to better understand how a project works in detail and why some decisions were taken. Considering this perspective, it is necessary to find an approach to organize and maintain knowledge about the decision making process from software development projects, and then use this knowledge to help people involved in these projects. Taking in account what has been stated above, this paper proposes an approach to document and organize the rationale regarding the decision making process in software development projects, using for this purpose a representation that encourages reasoning in order to define the best solutions for problems faced in software development project.

Key-words: Knowledge Management. Design Rationale. Decision Making.

\section{Introdução}

Um projeto de software envolve uma grande quantidade de conhecimento, o qual pode estar relacionado à tecnologia utilizada no projeto, à lógica de negócio necessária ao software, ou, ainda, estar relacionado a outras atividades ou dependências do projeto. Além disso, várias decisões são tomadas durante o desenvolvimento de um sistema e, mesmo após sua conclusão, em atividades de manutenção.

Considerando a grande quantidade de informação existente em projetos de software e o volume de decisões tomadas ao longo do seu desenvolvimento, é importante a existência de alguma ferramenta para documentar e manter estas decisões e, especialmente, os motivos pelos quais estas foram tomadas.

Esse tipo de documentação serve para que as pessoas envolvidas no projeto tenham uma visão mais ampla do mesmo e possam contribuir mais efetivamente, pois terão maior propriedade sobre seus detalhes. Isto é confirmado por Garvin [1], que afirma que saber "por que fazer" determinada atividade é fundamental, pois captura as relações de causa e efeito, admite exceções, adaptações e eventos inesperados. 
Outro fator a se considerar é que esta documentação pode ser útil para pessoas novas no projeto e, até mesmo, servir para reuso no futuro ou em outros projetos, o que, segundo Gupta et. al. [2], é o foco da Gestão do Conhecimento, ou seja, explicitar o conhecimento para que o mesmo possa ser compartilhado e reutilizado.

Dentro deste contexto, a Gestão do Conhecimento propõe a organização e o compartilhamento de todo o conhecimento importante a uma determinada atividade [2] [3] [4] [5] [6] [7]. De forma semelhante, abordagens de Design Rationale buscam manter, de forma estruturada e organizada, as decisões-chave tomadas durante o desenvolvimento de um projeto, para que estas possam ser úteis especialmente em atividades futuras [8].

Por estas razões, a pesquisa aqui apresentada visa aliar aspectos do Design Rationale à Gestão do Conhecimento e, a partir disto, obter uma representação que possibilite organizar e disponibilizar as principais decisões de projetos de software. A motivação para isso está no fato de que, de acordo com os conceitos de Gestão do Conhecimento e Design Rationale, manter as decisões e suas respectivas razões oferece maior vantagem competitiva a um projeto e possibilita maior troca de experiência até entre integrantes de diferentes projetos.

Assim, este artigo apresenta a especificação de uma técnica baseada em Design Rationale que visa contribuir para o gerenciamento do conhecimento relacionado ao processo de tomada de decisão em projetos de software. Através desta técnica, pretende-se representar, de forma simples e intuitiva, os problemas e alternativas ponderadas antes de cada decisão, reduzir a perda de capital intelectual quando pessoas saem do projeto, propiciar a outros projetos o reuso de experiências positivas e prevenir a repetição de problemas já vivenciados anteriormente.

Este trabalho está estruturado da seguinte forma: na seção 2 são apresentadas as abordagens de Design Rationale utilizadas como base para o desenvolvimento deste trabalho, a seção 3 aborda a análise do problema tratado e a seção 4 descreve a proposta elaborada. As seções 5 e 6 apresentam a aplicação da proposta e as considerações finais, respectivamente.

\section{Abordagens de Design Rationale Utilizadas}

Diferente do processo padrão de documentação, que consiste na descrição do resultado final de um projeto, a utilização de técnicas de Design Rationale busca documentar, além da decisão final, também as razões por trás de cada decisão, incluindo as justificativas, as alternativas consideradas e os argumentos que levaram a determinada decisão [8] [9].

Para Horner e Atwood [10], quando o Design Rationale é capturado e estruturado, o mesmo pode ser utilizado por pessoas que estão fora do contexto do projeto, a fim de analisarem artefatos e a influência das decisões tomadas durante o desenvolvimento do projeto. Assim, estas pessoas conseguem identificar os motivos pelos quais as decisões 
foram tomadas, bem como quais eram as alternativas consideradas e porque estas foram rejeitadas, evitando a repetição de trabalho já feito anteriormente.

Existem várias abordagens para representar Design Rationale e as utilizadas neste trabalho foram o QOC e a DRL:

- QOC (Questions, Options and Criteria): o QOC é baseado na formulação de questões, as quais são uma importante dimensão do espaço de projeto, representando os principais problemas que devem ser considerados, os quais compõem o espaço de alternativas. Critérios são objetivos positivos que servem como base para a avaliação e escolha entre as várias opções analisadas. E opções são as possíveis respostas às questões [11] [12]. Para a representação desta técnica, linhas contínuas representam relações positivas entre um critério e uma opção, enquanto linhas pontilhadas indicam uma relação negativa, ou seja, a opção não é suportada pelo critério. Um exemplo dessa representação é mostrado na Figura 1.

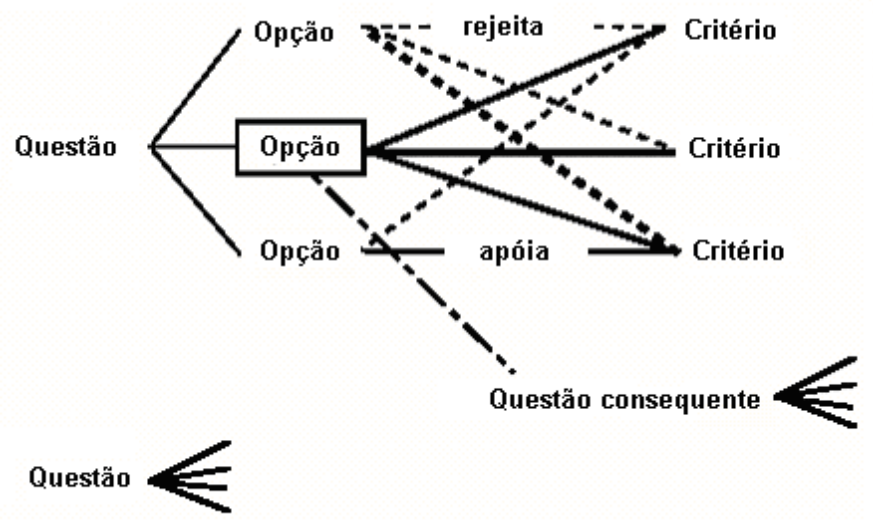

Figura 1. Exemplo genérico da notação QOC, adaptado de Shum e Hammond [13].

- DRL (Decision Representation Language): a DRL foi inicialmente desenvolvida como uma linguagem para representar o processo de tomada de decisão, sendo que posteriormente foi estendida para a representação de Design Rationale. Uma representação deve suportar certo número de tarefas de projeto, tais como, responder a questões sobre o progresso do projeto, as alternativas geradas, as avaliações que levaram a escolha de uma alternativa particular e a possível transferência de conhecimento para projetos futuros e outras pessoas [14]. A DRL tem como objetivo expressar estas questões. A 
Figura 2 exibe os elementos e relações que formam o vocabulário da linguagem DRL.

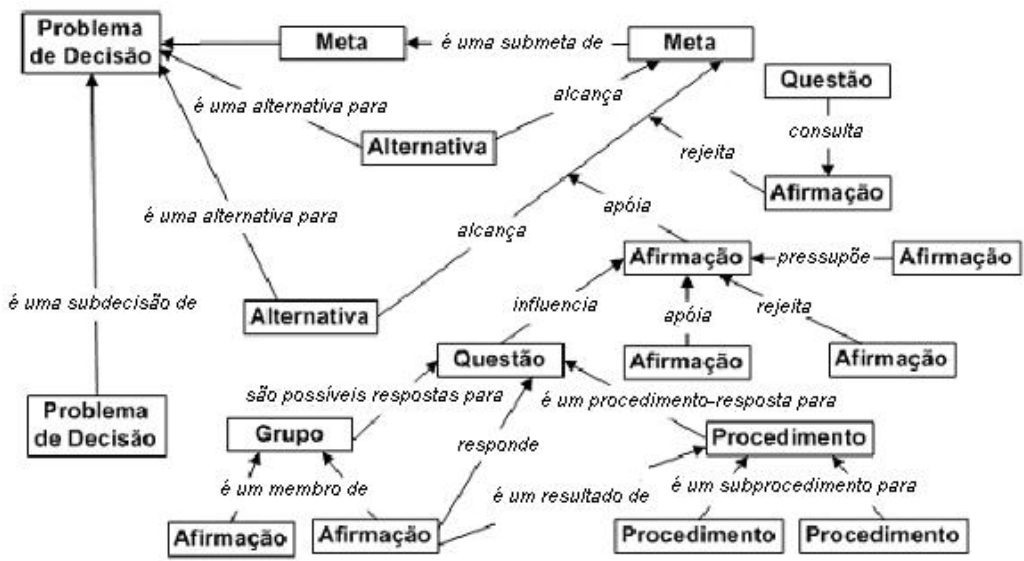

Figura 2. Vocabulário da linguagem DRL [15].

\section{Análise do Problema}

Com base nas necessidades e nos benefícios de se ter uma forma para manter as decisões de um projeto, partiu-se para a análise de algumas abordagens existentes, com o objetivo de identificar seus pontos fracos e fortes como método para registro de decisões. Em paralelo, foram pesquisadas as necessidades de profissionais que trabalham em projetos de software em termos de documentação de decisões para, assim, identificar, dentre as abordagens analisadas as características mais importantes e de maior aplicabilidade em projetos de software.

Como o interesse deste estudo era a captura das impressões dos participantes com relação às abordagens em estudo (e aos processos de construção e utilização das mesmas) e não uma medição em termos de quantidade, valor, intensidade ou freqüência [16], para a obtenção destes dados (opiniões, críticas e sugestões quanto às abordagens estudadas/propostas), foram realizados estudos qualitativos, com base em entrevistas com os participantes, a partir da análise das abordagens.

Assim, a partir da modelagem das decisões tomadas em um sistema real utilizando três abordagens diferentes (QOC, DRL e uma descrição textual livre), profissionais que atuam em projetos de desenvolvimento de software foram entrevistados com a intenção de se 
obter informações a respeito das modelagens apresentadas e a respeito de suas necessidades em termos de documentação de decisões importantes dentro de um projeto de software.

A seguir será descrito o sistema utilizado como base para a modelagem, bem como as entrevistas e a análise dos resultados obtidos.

\subsection{Descrição do Sistema Modelado}

O sistema utilizado neste estudo de caso foi elaborado por uma empresa que desenvolve soluções para a área de recursos humanos e folha de pagamento. O sistema em questão faz parte de um portal colaborativo e sua função é possibilitar a seleção de benefícios dinamicamente, ou seja, o objetivo do sistema é permitir que cada colaborador de determinada empresa tenha a possibilidade de escolher, dentre uma série de benefícios e coberturas disponíveis, aqueles benefícios que o interessam e satisfazem suas necessidades. Por exemplo, um colaborador de uma empresa deseja como benefício um plano de saúde para sua família, logo ele poderá optar dentre os planos de saúde disponíveis no sistema, aquele que oferece a cobertura para sua família e cujo custo mensal esteja de acordo com suas possibilidades.

Este sistema é integrado com outros sistemas já existentes, de onde provêm as informações sobre o colaborador, seus dependentes, benefícios e coberturas disponíveis. Sua função principal é oferecer uma interface integrada e um ponto de acesso único onde os usuários podem visualizar e atualizar informações e opções relacionadas a todos os benefícios oferecidos pela empresa em que trabalham.

\subsection{Entrevistas}

As entrevistas foram realizadas com um grupo de 8 profissionais que atuam em projetos de software, sendo 2 gerentes de projeto, 4 líderes de equipe e 2 analistas de sistemas. Todos os entrevistados possuem ampla vivência na área, com mais de 6 anos de experiência, inclusive com atuação em projetos internacionais. A escolha dos profissionais foi feita com base na função que desempenham dentro do projeto em que atuam, de forma a contar com a opinião de pessoas que participam do processo de tomada de decisões relativas ao projeto.

As entrevistas aconteceram através de uma discussão sobre a documentação das razões para as decisões tomadas em um projeto de maneira geral. Em seguida foram apresentadas as três abordagens utilizadas neste estudo. Para cada abordagem foi apresentada a representação conceitual da mesma, seguida da modelagem das decisões analisadas para o sistema de seleção de benefícios descrito anteriormente. Durante a discussão inicial, os entrevistados falaram sobre suas experiências relacionadas à documentação de projetos, sobre as carências que observam em seu dia-a-dia e sobre sua opinião pessoal a respeito do tema. 
Ao final, foi discutida a aplicabilidade destes modelos ou de modelos que tenham objetivos semelhantes e foram registradas as observações e sugestões de cada entrevistado. A partir destas informações foi possível compilar uma lista de observações e sugestões a serem analisadas e utilizadas como base para a proposta a ser elaborada.

\subsection{Análise dos Resultados}

Das abordagens utilizadas, o QOC foi a que teve maior aceitação pelos profissionais entrevistados, principalmente por possuir uma representação simples e de fácil entendimento e por instigar a busca de soluções a partir da definição dos principais problemas. A DRL foi considerada uma representação complexa e de difícil leitura, sendo mais suscetível à interpretação do que o QOC. Já a representação textual apresentada foi considerada interessante por poder ser bastante detalhada, porém é senso comum (e os profissionais também relataram isto) que as pessoas em geral não simpatizam com documentos longos, preferindo representações que sejam mais intuitivas e objetivas.

Com a realização das entrevistas, observaram-se, também, outros aspectos, que não estavam presentes nas abordagens apresentadas para os entrevistados, mas que foram considerados importantes e úteis se agregados a uma abordagem para representar as decisões de um projeto e suas razões. Entre estes aspectos, estão a representação da importância das questões e dos critérios favoráveis ou contrários a determinada opção, pois acredita-se que esta representação ajude a identificar os pontos críticos do projeto, que mereçam maior atenção, e as opções mais apropriadas para atender determinada questão. Outro aspecto considerado é a possibilidade de utilização de uma breve descrição textual dos principais itens da representação, como uma forma de contextualização para quem for utilizá-la posteriormente.

Com base nos resultados das entrevistas, optou-se pela utilização do QOC como ponto de partida para a proposta e, a partir disso, agregou-se, a esta técnica, características que objetivam tornar a abordagem mais completa e direcionada às necessidades de profissionais da área.

\section{QOC*}

Com base no estudo de caso realizado e no referencial teórico pesquisado, foi desenvolvida a proposta do QOC*. Sua notação é baseada no QOC, cuja representação é simples e não possui muitas relações e possibilidades de representação, sendo composta por questões, opções, critérios e relações positivas ou negativas entre as opções e os critérios.

Além disso, esta proposta introduz novos itens:

- Representação da importância das questões: as questões são os itens mais importantes da modelagem baseada no QOC, pois é a partir delas que é desencadeado todo o processo de modelagem. A identificação de boas 
questões é crítica, porque tais questões podem reestruturar a maneira como os problemas são vistos e definir onde buscar as soluções para estes problemas [17]. Com base nisto introduziu-se a representação da importância das questões, pois se acredita que isto facilite a leitura da representação e permita verificar os pontos críticos do projeto e suas decisões relacionadas. Para simbolizar esta importância de forma diagramática, as questões passam a ser representadas por caixas com bordas arredondadas, sendo que caixas com borda simples representam uma questão de importância moderada; com borda dupla representam uma questão de importância média; e com borda tripla representam uma questão de importância elevada.

- Representação do peso dos critérios: de forma semelhante às questões, os critérios também passaram a ter uma representação de importância, cujo objetivo é facilitar a identificação de quais opções são mais apropriadas para resolver determinada questão. A utilização de pesos surge como uma ferramenta para ajudar a identificar, dentre os critérios estabelecidos para uma decisão, aqueles que são mais significativos. Sendo assim, introduziu-se a representação do peso de um critério através do uso de asteriscos (*), de forma que: critério sem asterisco representa um critério normal, com relevância moderada; com um asterisco (*) representa um critério importante com relevância média; e com dois asteriscos (**) representa um critério muito importante e altamente relevante.

- Descrição textual: a opção por uma representação diagramática baseia-se no fato de que esta é uma forma mais objetiva e compacta de representação, que ao mesmo tempo possibilita uma visão mais ampla do projeto. Porém, como complemento, pode ser adicionada, a esta, uma descrição textual sucinta que visa explicar a representação dos diagramas. A grande razão para se ter essa descrição é oferecer uma forma de contextualização para quem está analisando a representação e as decisões tomadas, pois somente a representação diagramática pode dificultar a percepção do contexto. Basicamente, o complemento textual consiste em uma breve descrição das Questões e Critérios classificados com relevância alta ou média.

A Figura 3 exibe a representação conceitual do diagrama utilizando a representação da importância das questões e os pesos para os critérios. 


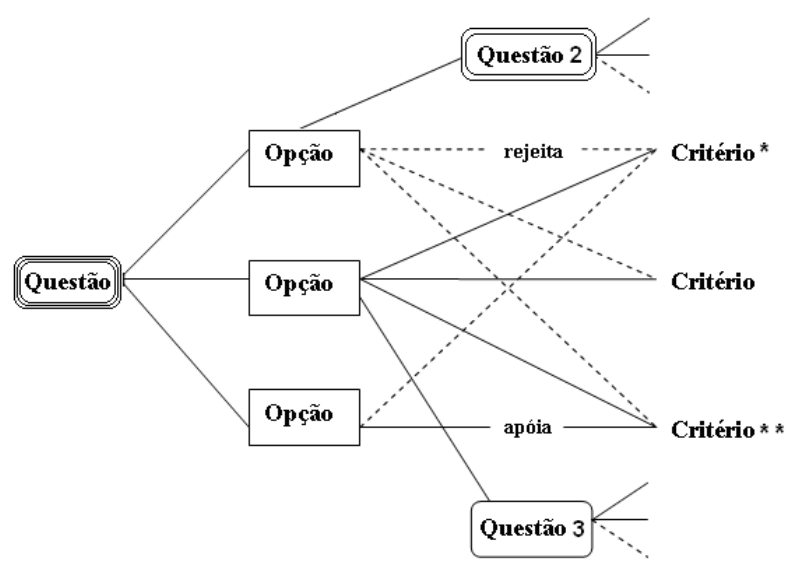

Figura 3: Representação conceitual da importância das questões e pesos dos critérios.

\section{Aplicação da Proposta}

A fim de discutir a aplicabilidade e relevância da abordagem proposta, realizou-se um estudo considerando a realidade de um projeto de software. Para isto contou-se com a participação de profissionais de mercado, que participam das decisões tomadas nos projetos em que atuam, a fim de obter feedback de representantes do público alvo da abordagem QOC**.

\subsection{Perfil dos Participantes}

O estudo foi realizado com 10 pessoas $^{3}$, com diferentes atuações em projetos de software. Informações sobre suas áreas de atuação, bem como sua participação no processo de decisão de projetos e o uso de técnicas para documentação de decisões, são apresentadas na Tabela 1 .

\footnotetext{
${ }^{3}$ A seleção de pessoas que atendessem ao perfil desejado e que pudessem participar do experimento foi um obstáculo a ser vencido. De um total de 35 pessoas convidadas, apenas 10 tiveram disponibilidade para participar da pesquisa
} 
Tabela 1. Informações sobre os participantes.

\begin{tabular}{|c|c|c|c|c|c|c|c|c|}
\hline \multirow{2}{*}{$\begin{array}{l}\text { Partici- } \\
\text { pante }\end{array}$} & \multirow[b]{2}{*}{ Idade } & \multicolumn{5}{|c|}{ Áreas de atuação (experiência em anos) } & \multirow{2}{*}{$\begin{array}{l}\text { Participa no } \\
\text { processo de } \\
\text { decisão }\end{array}$} & \multirow{2}{*}{$\begin{array}{c}\text { Documenta } \\
\text { decisões }\end{array}$} \\
\hline & & Gerência & $\begin{array}{c}\text { Arqui- } \\
\text { tetura }\end{array}$ & $\begin{array}{c}\text { Desenvol- } \\
\text { vimento }\end{array}$ & $\begin{array}{c}\text { Análise/ } \\
\text { Projeto }\end{array}$ & Teste & & \\
\hline 1 & 26 & 2 & & 8 & 4 & & Diretamente & Sim \\
\hline 2 & 41 & 10 & & 15 & 15 & & Diretamente & Não \\
\hline 3 & 30 & & 4 & 9 & 7 & & Diretamente & Não \\
\hline 4 & 34 & 8 & & & & & Indiretamente & Não \\
\hline 5 & 26 & 1,5 & & & & 7 & Diretamente & Sim \\
\hline 6 & 27 & 3 & 1 & & & 7 & Diretamente & Sim \\
\hline 7 & 26 & & & 4 & 2 & & Indiretamente & Não \\
\hline 8 & 28 & 5 & & 6 & 6 & 7 & Diretamente & Sim \\
\hline 9 & 34 & 1 & & 10 & 10 & & Diretamente & Sim \\
\hline 10 & 32 & 9 & & 20 & 12 & & Indiretamente & Não \\
\hline
\end{tabular}

Pode-se observar que a faixa etária dos participantes variou entre 26 e 41 anos, o que caracteriza uma população com uma grande diversidade de conhecimento e experiência em projetos de software, seja em termos de tecnologias utilizadas ou metodologia de gerenciamento de projetos. Acredita-se que este fato contribuiu para uma maior riqueza no resultado do experimento, pois são pessoas com experiências bem diferenciadas.

Outro fato a ser considerado é que metade dos participantes disse nunca ter usado nenhuma técnica para documentar decisões. Da outra metade, que já utilizou, a maioria mencionou atas de reuniões ou e-mails para documentação, sendo que ainda foram citadas outras técnicas como mapas mentais (mind maps) e diagramas espinha de peixe, além de outros modelos específicos a determinados projetos.

Ao mesmo tempo em que estas informações demonstram que não é muito comum se documentar decisões (metade da população observada nunca utilizou nenhuma técnica para isso), também é possível perceber que existe a preocupação de se manter estas informações com, inclusive, iniciativas neste sentido (seja com documentação puramente textual como atas e $e$-mails, seja com a utilização de algum tipo de representação).

\subsection{Dinâmica}

Em termos gerais, os participantes deveriam modelar as decisões envolvidas em um sistema de software fictício, utilizando a abordagem QOC*. O objeto da modelagem foi um sistema para gerenciamento de projetos de pesquisa acadêmicos, composto por três módulos: 1) Gerência de RH; 2) Gerência de Recursos financeiros e imobilizados; 3) Gerência dos projetos acadêmicos.

Como material de apoio, foi preparado um roteiro contendo a contextualização do assunto, a descrição da abordagem QOC*, um exemplo de modelagem com QOC*, a descrição do sistema para o qual deveria ser feita a análise das possíveis decisões utilizando a nova abordagem e um questionário para avaliar a experiência proporcionada ao usuário e as dificuldades relacionadas ao desenvolvimento da modelagem. 
Devido à dificuldade para agendar horário com todos os participantes, dado suas ocupações profissionais, optou-se por duas modalidades para realização do estudo: 1) com monitoramento, em que os participantes foram assistidos e tiveram liberdade para questionar e tirar dúvidas durante a realização da modelagem; 2) individual, em que o participante realizou a modelagem sozinho, sem assistência, apenas baseado no documento com o roteiro.

Um ponto interessante da segunda opção foi justamente a possibilidade de verificar a dificuldade no entendimento da proposta e no desenvolvimento da modelagem, visto que apenas uma descrição textual foi oferecida.

De maneira geral, a realização do experimento propiciou a obtenção de informações importantes sobre a abordagem proposta, como aplicabilidade da mesma, dificuldade de entendimento, além de vantagens da sua utilização, as quais serão apresentadas na próxima seção.

\subsection{Discussão}

A Tabela 2 apresenta um sumário das informações obtidas com o experimento, sendo que estas são comentadas na seqüência.

Tabela 2. Resultados do experimento.

\begin{tabular}{|c|c|c|c|c|}
\hline Participante & $\begin{array}{c}\text { Modalidade de } \\
\text { execução }\end{array}$ & Duração (min) & $\begin{array}{c}\text { Nível de } \\
\text { dificuldade } \\
\text { da proposta }\end{array}$ & $\begin{array}{c}\text { Fases de um } \\
\text { projeto } \\
\text { na qual é útil }\end{array}$ \\
\hline $\mathbf{1}$ & Individual & 35 & Baixo & Análise e Projeto \\
\hline $\mathbf{2}$ & Individual & 80 & Baixo & Análise e Projeto \\
\hline $\mathbf{3}$ & Monitorado & 40 & Baixo & Análise e Projeto \\
\hline $\mathbf{4}$ & Monitorado & 30 & Médio & Análise \\
\hline $\mathbf{5}$ & Monitorado & 120 & Alto & Análise \\
\hline $\mathbf{6}$ & Individual & 50 & Baixo & Análise \\
\hline $\mathbf{7}$ & Monitorado & 40 & Baixo & Análise e Projeto \\
\hline $\mathbf{8}$ & Individual & 45 & Baixo & Análise \\
\hline $\mathbf{9}$ & Individual & 60 & Médio & Análise \\
\hline $\mathbf{1 0}$ & Individual & 50 & & \\
\hline
\end{tabular}

Um ponto observado na realização do experimento foi o tempo de duração para cada participante, ou seja, quanto tempo cada pessoa levou para entender a técnica e realizar a modelagem das decisões envolvidas no sistema fictício apresentado. O tempo médio necessário foi de 55 minutos, sendo o maior tempo de duração, 2 horas e o menor, 35 minutos. É importante destacar que o sistema apresentado para ser modelado é relativamente 
simples, pois o objetivo não era o desenvolvimento de uma modelagem complexa, mas, sim, identificar a dificuldade de entendimento e aplicação da abordagem proposta.

Com relação ao entendimento da abordagem e da notação do QOC*, $90 \%$ dos entrevistados consideraram de médio a fácil, achando os elementos bem definidos, intuitivos e expressivos. Em dois casos houve relato de dificuldade de compreensão da abordagem em um primeiro momento, sendo que esta dificuldade acabou sendo superada durante a modelagem.

Sobre a aplicabilidade da proposta em projetos de software, todos os entrevistados consideram a mesma aplicável, principalmente pelo fato da abordagem instigar o levantamento de questões e a busca de opções para atender estas questões, o que incentiva a discussão. Ao mesmo tempo, a abordagem incentiva a formalização do que está sendo discutido, proporcionando, desta forma, um histórico das questões e das opções analisadas, bem como dos critérios que levam a aceitação ou não de determinada opção.

No entanto, percebeu-se certa preocupação com relação à utilização da abordagem proposta em projetos grandes e complexos, no sentido de que a representação possa se tornar confusa e muito complexa, o que dificultaria o entendimento da modelagem e conseqüentemente sua utilização. Além disso, foi levantada a necessidade de uma ferramenta para ajudar na aplicação do QOC*, de forma a facilitar a criação dos diagramas e possibilitar uma forma padronizada de representação, bem como possibilitar a organização e a recuperação das modelagens [18].

Além da aplicabilidade da abordagem, os participantes do experimento foram questionados a respeito da fase do projeto em que este tipo de modelagem melhor se adequaria, sendo que, por unanimidade, foi citada a fase inicial de um projeto, ou seja, a fase de investigação e análise de requisitos. Além desta, outras fases também foram citadas, como o planejamento da arquitetura e o projeto do software.

Uma consideração levantada durante o experimento, e que também é considerada e destacada neste trabalho, é que esta abordagem deve ser utilizada nas principais e mais importantes decisões de um projeto, e não em toda e qualquer decisão. O uso de forma exaustiva da proposta seria bastante difícil considerando a realidade de um projeto de desenvolvimento de software.

A partir das entrevistas, observou-se como principais vantagens da proposta apresentada a possibilidade de se manter e reaproveitar os problemas identificados e as opções avaliadas em projetos de software, pois estas informações são úteis e importantes para a continuidade de um projeto. Outro aspecto a se considerar é que essas informações podem, também, vir a ser utilizadas em outros projetos, pois muitas decisões acontecem diante de cenários recorrentes, logo, o histórico das decisões e suas razões, desde que acessível, pode ser útil para gerenciar o conhecimento existente em um projeto [19] [20].

Além da necessidade de uma ferramenta para realizar a modelagem utilizando o QOC*, observou-se a necessidade de uma ferramenta que permita a pesquisa em uma base de conhecimento que contenha as informações sobre as decisões de diversos projetos e 
possibilite o relacionamento entre estas informações, automatizando assim o processo de reaproveitamento das informações armazenadas.

De maneira geral, acredita-se que o resultado da utilização do QOC* foi positivo, considerando-se que as pessoas conseguiram entender a abordagem apresentada com relativa facilidade e conseguiram, também, vislumbrar pontos positivos de sua utilização nos projetos em que trabalham. Como uma proposta nova, com certeza há muitos pontos que podem ser aprimorados, tornando-a, assim, mais atraente e completa.

É importante também considerar que existem várias técnicas, com propósitos diferentes, que podem ser utilizadas em projetos de software. Devido a natureza destes projetos, muitas vezes a inserção de novas técnicas é um desafio, pois provoca um impacto no projeto, o que nem sempre é aceitável.

\subsection{Refinamento da Proposta}

Após a realização do experimento e a análise dos resultados, notou-se que algumas características ainda poderiam ser alteradas ou adicionadas na proposta, com a intenção de torná-la mais interessante e completa:

- descrição dos itens: julgou-se interessante também descrever - sucintamente - as opções analisadas, além de todas as questões e critérios. Através da descrição textual dos itens que compõe a modelagem, acredita-se que a contextualização do projeto fique mais completa e facilite a leitura da representação diagramática;

- decisões a documentar: é inviável avaliar e representar todo tipo de questão envolvida em um projeto de software, pois isso geraria uma complexidade muito grande e tornaria impossível armazenar e consultar as informações relacionadas às decisões tomadas. Assim, para efeitos de representação, é necessário considerar somente as questões mais importantes de todo o projeto, cujo impacto e risco são relevantes e cujas decisões caracterizam determinado projeto. Exemplos de decisões consideradas relevantes através do estudo e das entrevistas realizadas foram: decisões sobre regras de negócio; decisões de projeto/ arquitetura do software; decisões sobre a arquitetura de desenvolvimento do projeto; decisões que impactam e ameaçam o cronograma do projeto.

- destaque da escolha: apesar da abordagem em questão ter como objetivo manter tanto as decisões tomadas quanto às opções que deixaram de ser escolhidas por determinada razão, é importante conseguir-se identificar qual das alternativas avaliadas foram realmente escolhidas. Para isso, optou-se por representar a opção escolhida através de uma caixa preenchida, diferente das outras opções que utilizam caixa sem preenchimento, destacando assim as opções selecionadas e aplicadas no projeto. 


\section{Considerações Finais}

Considerando-se a grande quantidade de conhecimento necessário e existente em projetos de software, o presente trabalho apresenta uma proposta para representar o conhecimento envolvido no processo de tomada de decisão deste tipo de projeto. Esta proposta abrange a representação não somente das alternativas escolhidas, mas sim de todas as alternativas avaliadas para resolver os principais problemas de decisão presentes em projetos de desenvolvimento de software. A representação proposta pelo QOC*, baseada na abordagem QOC, estimula a definição das principais questões existentes em um projeto e a análise das alternativas que possam vir a ser escolhidas para suprir as necessidades levantadas em cada questão.

A fim de compreender melhor o problema em questão, foram estudadas e aplicadas técnicas de Design Rationale em um projeto de software. A partir disto, foram realizadas entrevistas com profissionais que atuam em projetos de desenvolvimento de software, com o objetivo de identificar necessidades destes profissionais em termos de documentação das decisões tomadas ao longo do projeto. Durante as entrevistas, foram apresentadas algumas possibilidades de documentação (com exemplos), e discutiu-se a aplicabilidade destas no dia-a-dia de um projeto. Foram analisadas, também, várias considerações feitas pelos entrevistados, com relação às carências e às sugestões para documentar as decisões e as razões das decisões de projeto.

A partir da análise do resultado das entrevistas, elaborou-se uma proposta baseada em Design Rationale, a qual agrega algumas das sugestões feitas pelos entrevistados e aspectos observados nos estudos realizados. Assim que a proposta foi definida, foi necessária novamente a participação de profissionais para ajudar a validar a mesma. Nesta ocasião os participantes tiveram que modelar as decisões envolvidas em um sistema fictício utilizando a abordagem QOC*, e então responder a um questionário, cujo objetivo era obter o feedback dos participantes a respeito da proposta.

Uma das principais razões pela qual a presente proposta foi desenvolvida com base no QOC é justamente a facilidade de entendimento e representação desta abordagem, confirmada nas entrevistas realizadas. Apesar de não existir uma ferramenta computacional destinada especificamente para este tipo de representação, é relativamente fácil representá-la manualmente ou, então, com o auxílio de algumas ferramentas existentes como o MSWord® ou o MSVisio®, entre outras.

Por fim, um aspecto observado, é que a atividade de desenvolvimento de software é uma atividade diretamente dependente do conhecimento, de forma que muitas vezes este conhecimento está concentrado em apenas algumas pessoas; logo, a saída de um colaborador chave pode significar um impacto muito grande no projeto. Através da utilização de abordagens como o QOC* é possível manter-se aspectos chave do projeto e, principalmente, o porquê destes aspectos, o que com certeza facilita a disseminação do conhecimento, a curva de aprendizagem de um novo colaborador e o entendimento global do projeto. 


\section{Referências}

[1] GARVIN, D. A. Construindo a Organização que Aprende, In: Gestão do Conhecimento (Harvard Business Review on Knowledge Management), pp 50-81, $4^{\text {a }}$ ed., Campus, Rio de Janeiro 2000.

[2] GUPTA, B.; IYER, L. S.; ARONSON, J. E. Knowledge Management: Practices and Challenges, Industrial Management and Data Systems, vol.100, pp 17-21, nº 1, 2000.

[3] DAVENPORT, T.; PRUSAK, L. Conhecimento Empresarial: como as organizações gerenciam seu capital intelectual, Rio de Janeiro: Campus, $4^{\mathrm{a}}$ edição, 1998. 237 p.

[4] LAWTON, G. Knowledge Management: Ready for Prime Time?, Computer, vol. 34, pp. 12-14, n 2, 2001.

[5] NONAKA, I. "The Knowledge Creating Company", Harvard Business Review, vol. 69, pp 96-104, nº 6, 1991.

[6] SIVAN, Y.Y. Nine Keys to a Knowledge Infrastructure: A Proposed Analytic Framework for Organizational Knowledge Management. Harward University, March 2001.

[7] ZACK, M.H. Developing a Knowledge Strategy, California Management Review, vol. 41, pp 125-145, $\mathrm{n}^{\circ}$ 3,1999.

[8] MORAN, T. P.; CARROLL, J. M.; LEE J.; LAI, K. Overview of Design Rationale, In 'Design Rationale: Concepts, techniques, and Use', Lawrence Erlbaum associates, Mahwah, NJ, 1996.

[9] LEE, J. Design Rationale Systems: Understanding the Issues, IEEE Expert, Vol. 12, pp. 78-85, no 3, May 1997.

[10] HORNER, J.; ATWOOD, M. E. Design rationale: the rationale and the barriers. In: Proceedings of the 4th Nordic Conference on Human-computer interaction, Oslo, Norway, pp 341-350, October, 2006.

[11] PREECE, J.; ROGERS, Y.; SHARP, H.; BENYON, D.; HOLLAND, S.; CAREY, T. Human-Computer Interaction, Addison-Wesley, Wokingham, UK, 1994.

[12] MACLEAN, A.; YOUNG, R. M.; BELLOTTI, V.; MORAN, T., Questions, Options, and Criteria: Elements of Design Space Analysis. In: Design Rationale: Concepts, Techniques, and Use, Lawrence Erlbaum associates, Mahwah, NJ, 1996.

[13] SHUM, S. B.; HAMMOND, N. Argumentation-Based Design Rationale: What Use at What Cost?, International Journal of Human-Computer Studies, vol 40, pp 603-652, nº 4, April 1994.

[14] LEE J; LAI, K. What's in Design Rationale? In: Design Rationale: Concepts, techniques, and Use, Lawrence Erlbaum associates, Mahwah, NJ, 1996

[15] MEDEIROS, A. P. Kuaba: Uma Abordagem para Representação de Design Rationale para o Reuso de Designs baseado em Modelos, Pontifícia Universidade Católica do Rio de Janeiro, 149 f., Tese de Doutorado, Rio de Janeiro, Março, 2006.

[16] DENZIN, N. K.; LINCOLN, Y. S., The landscape of qualitative research: Theories and issues, Sage Publications Inc., Thousand Oaks, CA, 2003.

[17] BELLOTTI, V.; MACLEAN, A.; and MORAN, T. What makes a Good Design Question? SIGCHI Bulletin, vol. 23, pp 80-81, no 4, October 1991.

[18] BRACEWELL, R.; WALlACE, K.; MOSS, M.; KNOTT, D., Capturing design rationale, Computer-Aided Design, vol 41, pp 173-186, March 2009.

[19 ] FALESSI, D.; CANTONE, G.; BECKER, M., Documenting design decision rationale to improve individual and team design decision making: an experimental evaluation. In Proceedings of the 2006 ACM/IEEE international Symposium on Empirical Software Engineering, Rio de Janeiro, Brasil, September, 2006.

[20] BURGE, J. E., Design rationale: Researching under uncertainty. Artif. Intell. Eng. Des. Anal. Manuf., vol 22, pp311-324, November 2008 . 